\title{
REGISTRO DE PALEONISCÓIDES NA BASE DO MEMBRO TAQUARAL, FORMAÇÃO IRATI, PERMIANO DA BACIA DO PARANÁ
}

\author{
Artur CHAHUD \\ Setembrino PETRI
}

\begin{abstract}
RESUMO
Uma camada de arenito conglomerático localizada na base da Formação Irati (Grupo Passa Dois) no município de Rio Claro, centro-leste do Estado de São Paulo, Brasil, exibe diversas espécies de vertebrados fósseis. Ocorrem formas de Chondrichthyes representadas por dentes e espinhos de diversas ordens, mas são os Osteichthyes os fósseis mais abundantes, caracterizados por escamas, dentes e fragmentos ósseos indeterminados de Actinopterygii. São descritos aqui dentes e escamas deste grupo encontrados na base do Membro Taquaral da Formação Irati.
\end{abstract}

Palavras-chave: Paleoniscóides, Formação Irati, Membro Taquaral, Rio Claro, Permiano.

\begin{abstract}
A conglomeratic sandstone, the lowest bed of the Taquaral Member, the basal unit of the Permian Irati Formation (Passa Dois Group), in the Municipality of Rio Claro, east-central State of São Paulo, Brazil, contains several species of vertebrate fossils. Chondrichthyes of different orders are represented by teeth and spines, but the most abundant fossils belong to the Osteichthyes as represented by paleoniscoid scales, teeth and bone fragments. The Actinopterygii are the commonest fish remains in this unit.
\end{abstract}

Keywords: Paleoniscoids, Irati Formation, Taquaral Member, Rio Claro, Permian.

\section{INTRODUÇÃO}

A região centro-leste do Estado de São Paulo expõe praticamente toda a seção neopaleozóicamesozóica da borda leste da Bacia do Paraná, começando com as unidades neopaleozóicas do Grupo Tubarão (Subgrupo Itararé e Formação Tatuí), passando pelo Grupo Passa Dois (formações Irati e Corumbataí) e terminando com as formações mesozóicas do Grupo São Bento (formações Pirambóia, Botucatu e Serra Geral). Em discordância sobre estas rochas ocorrem depósitos cenozóicos da Formação Rio Claro e coberturas de colúvio e aluvião neógenas.

Em discordância sobre a Formação Tatuí ocorrem sedimentos relativamente grossos, arenitos com grânulos e arenitos conglomeráticos passando para conglomerados em contato abrupto, acima, com folhelhos síltico-argilosos do Membro
Taquaral, base da Formação Irati (RAGONHA 1978, FÚLFARO et al. 1984, STEVAUX et al. 1986, HACHIRO 1997, ASSINE et al. 2003).

Os paleovertebrados da base da Formação Irati receberam pouca atenção até agora e nunca foram analisados dentro da Estratigrafia. Estudos recentes (CHAHUD 2007) revelaram surpreendente diversidade taxonômica desta paleoictiofauna.

Atualmente, a Formação Irati é considerada de idade eopermiana, a partir de dados isotópicos recentemente obtidos por SANTOS et al. (2006), em torno de $278 \mathrm{Ma} \pm 2,2 \mathrm{Ma}$, Artinskiano (Cisuraliano). Apesar de obtida na região sul do Brasil, cidade de São Mateus do Sul, no Estado do Paraná, é semelhante às idades dos sedimentos de posições estratigráficas equivalentes na África do Sul, podendo ser aplicadas às rochas encontradas em São Paulo.

O objetivo deste artigo é a apresentação de diferentes morfotipos de paleoniscóides encontrados 
na base do Membro Taquaral da Formação Irati, no Sítio Santa Maria, na divisa entre os municípios de Rio Claro e Ipeúna. Eles foram comparados com outros fósseis encontrados no Permo-Carbonífero da Bacia do Paraná, acrescentando-se assim, mais uma localidade para o estudo de Osteichthyes fósseis do Neopaleozóico.

Os peixes paleoniscóides (ou "Palaeonisciformes") formam um grupo parafilético de actinopterígeos basais do Paleozóico. Segundo RICHTER et al. (2004), são representativos de sete famílias, com parentescos duvidosos, de grande sucesso no Paleozóico. O grupo continuou durante o Mesozóico com poucos representantes até o Cretáceo (FIGUEIREDO \& GALLO 2006a, b).

As escamas nos Paleoniscóides são do tipo ganóide com formato romboédrico, constituição rígida. Apresentam uma camada basal de osso lamelar celular, uma camada de dentina (ortodentina) e uma cobertura de esmalte de origem ectodérmica (ganoína) (RICHTER et al. 2004, FIGUEIREDO \& GALLO 2006a).

Os dentes consistem, basicamente, de uma fuste de formato cônico com um capuz apical maciço, composto de acrodina (um enameloide). A superfície da fuste pode ser lisa ou ornamentada por costelas ou pequenos tubérculos. São constituídos, internamente, por ortodentina com uma camada de esmalte em volta (colar de ganoína) (RICHTER et al. 2004, FIGUEIREDO \& GALLO 2006a).

\section{MATERIAL E MÉTODOS}

A pesquisa foi realizada em afloramentos do Sítio Santa Maria na região de Rio Claro (Figura 1), sempre em camadas sedimentares da base da Formação Irati. A escolha da área e do intervalo estratigráfico de estudo foi motivada pela abundância de afloramentos fossilíferos e pela disponibilidade de dados geológicos. A região de estudo é bem servida por estradas, na maioria não pavimentadas, e tem como acesso as rodovias SP-191, SP310 e SP-330.

Os restos de paleoniscídeos são os mais abundantes em toda a coleção de fósseis estudados. O material coletado consiste de escamas, dentes isolados e partes ósseas.

Os fósseis, em geral, são pequenos e fortemente cimentados à matriz de arenitos finos a muito finos. Muitas das melhores peças esqueléticas foram retiradas da matriz sob microscópio estereoscópico com ajuda de agulhas e pinças. Foram obtidas imagens digitais em um Omega Megascan
- ACCU 6000 Scanner, uma máquina fotográfica analógica SONY 3CCD e processadas em um analisador de imagem da LEICA do Laboratório de Petrografia Sedimentar do Instituto de Geociências da Universidade de São Paulo (IGc-USP). Todos os espécimes estão registrados e depositados na coleção fóssil de vertebrados $(\mathrm{GP} / 2 \mathrm{E})$ do Laboratório de Paleontologia Sistemática (LPS) do IGc-USP.

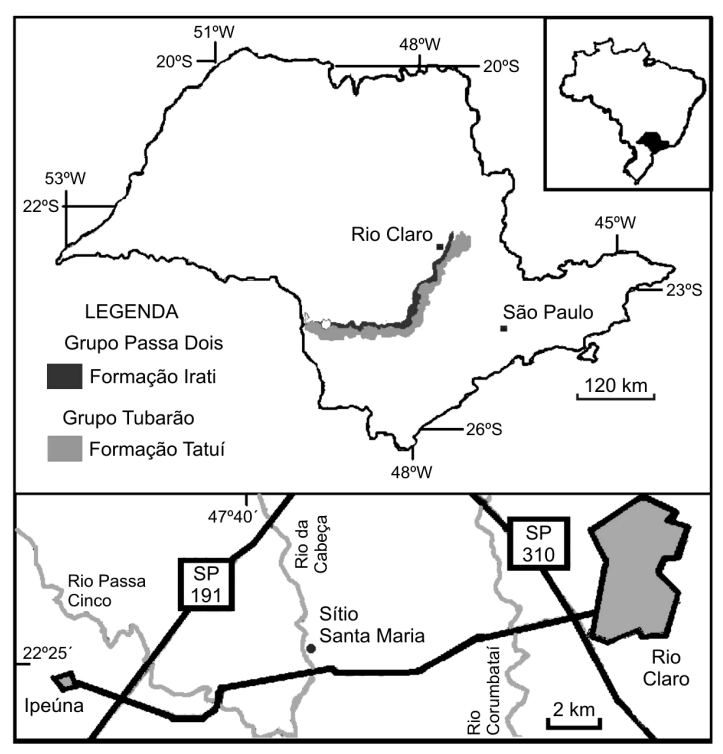

FIGURA 1 - Mapa do Estado de São Paulo destacando as faixas de ocorrência das formações Tatuí e Irati. Abaixo, mapa local destacando as principais rodovias de acesso ao Sítio Santa Maria.

Apesar da abundância de paleoniscóides, a identificação de espécies somente é possível em exemplares completos ou semicompletos, dada a baixa variação morfológica externa entre os mais diversos gêneros. RICHTER et al. (1985) ainda conseguiram associar diversas escamas isoladas a determinadas espécies. Os mais antigos peixes desse grupo, do Eopaleozóico, também foram diferenciados com base em escamas (LONG 1993, 1995).

\section{DESCRIÇÃO DE ICTIODONTES}

3.1 Ictiodontes de fuste lisa

Material - GP/2E-5941, GP/2E-5942. Dentes isolados.

Descrição - Dentes alongados, cônicos, com fuste lisa (Figuras 2A e B), de coloração mais clara nos exemplares em bom estado de preservação. 
A fuste normalmente não é robusta e em alguns exemplares a superfície está desgastada. É o menor tipo de todos os dentes estudados, variando em comprimento entre 0,5 e 2,0 mm. Os dentes mais comuns são retos, porém existem formas curvas.

\subsection{Ictiodontes retos de fuste costelada}

Material - GP/2E-5948. Dente isolado.

Descrição - Os dentes são alongados, cônicos e com a fuste ornamentada por costelas (Figuras $2 \mathrm{C}$ e D). O capuz apical, quando presente, é liso e maciço. As costelas normalmente são homogêneas da base até o capuz apical, porém em um exemplar, GP/2E-5948, as costelas são mais proeminentes na base e vão se afilando, tornando-se menos nítidas próximo ao capuz apical. A fuste, em geral, é alongada, formando um cone de ângulo agudo. Em alguns exemplares a fuste é robusta. São dentes pequenos, com comprimento inferior a $2 \mathrm{~mm}$.

3.3 Ictiodontes curvos ou sigmoidais de fuste costelada

Material - GP/2E-5949, GP/2E-5950 e GP/2E-5973. Dentes isolados.

Descrição - De todos os dentes de paleoniscóides, esta é a variedade mais comum. Os dentes são alongados e curvos (Figuras 2E e F) ou com forma sigmóide. O caráter sigmóide só se revela na base do dente e apenas em exemplares maiores e bem preservados. A fuste é ornamentada por costelas e normalmente não é muito robusta, pois na maioria dos dentes o diâmetro da base equivale a 1/5 do seu comprimento. O capuz apical é liso, alongado e mais claro que a fuste.

O comprimento varia entre 2 e $30 \mathrm{~mm}$. As costelas variam, desde relativamente espessas, em exemplares menores que $1 \mathrm{~mm}$, a finas e pouco espaçadas em exemplares com mais de $1 \mathrm{~cm}$ de comprimento.

\subsection{Discussão dos exemplares}

A maioria dos dentes paleoniscóides da base da Formação Irati é idêntica aos estudados por WÜRDIG-MACIEL (1975) e RICHTER et al. (1985) na Formação Estrada Nova, Grupo Passa Dois, Rio Grande do Sul. Nestes trabalhos os dentes foram separados em 10 categorias morfológicas com base na ornamentação e curvatura, I-1 a I-10 (RICHTER et al. 1985). Entretanto, ao contrário dos exemplares da região sul, que praticamente não exibem indícios de abrasão, os fósseis da base da Formação Irati sofreram desgaste superficial e quebras, o que dificulta a diferenciação - especialmente, entre dentes sigmóides e curvos (I-3 e I-4 são indistinguíveis de I-6 e I-7, respectivamente) - e, também, a constatação de micro-tubérculos na fuste (I-3 e I-4 semelhantes a I-9 e I-10), limitando a comparação.

Apesar do diferente grau de preservação, apenas dois tipos de ictiodontes descritos por RICHTER et al (1985), I-5 e I-8, na região sul do Brasil não foram observados nos da Formação Irati. Por outro lado, dois tipos descritos no presente trabalho diferem dos encontrados no Neopermiano
A

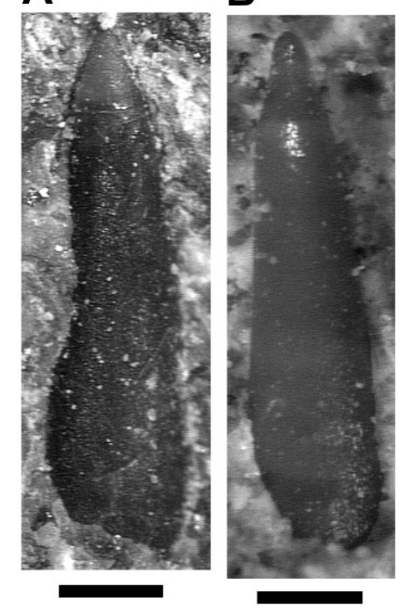

C

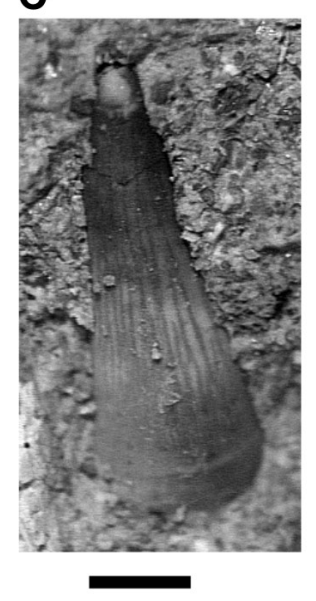

D

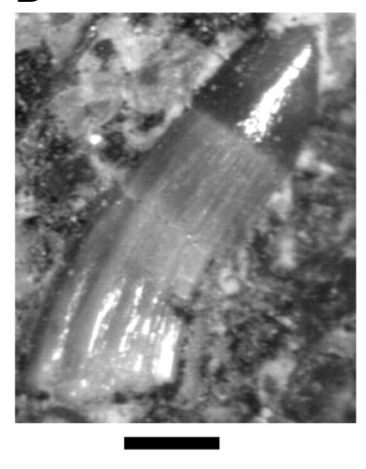

E

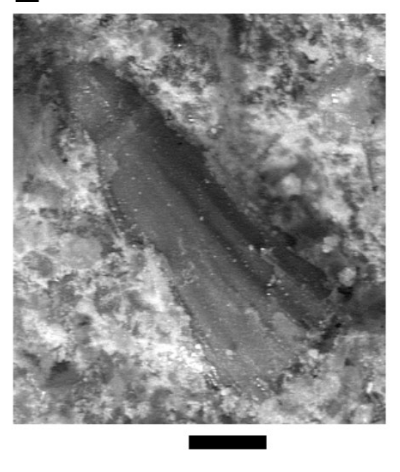

FIGURA 2 - Ictiodontes. A-B: dentes lisos (GP/2E-5941, GP/2E-5942). C: dente reto costelado (GP/2E-5948) e D-E: dentes curvos costelados (GP/2E-5949, GP/2E-5950). Barra de escala 0,2 mm. 
da região sul, os grandes dentes curvos e costelados com mais de um centímetro e um dente costelado apenas na base.

Os dentes menores que $1 \mathrm{~mm}$ são muito semelhantes aos exemplares descritos no Neopermiano da região sul e em outras partes da Bacia do Paraná (como os da Formação Corumbataí, MARANHÃO 1995), porém os de tamanho centimétrico (Figura 3) se assemelham ao dente descrito na fácies Budó (Subgrupo Itararé) - Rio Grande do Sul (BARCELLOS 1975) e a dentes da Formação Pedra do Fogo (COX \& HUTCHINSON 1991). Estes grandes dentes aparentemente pertenceram aos maiores paleoniscóides que habitaram a região.

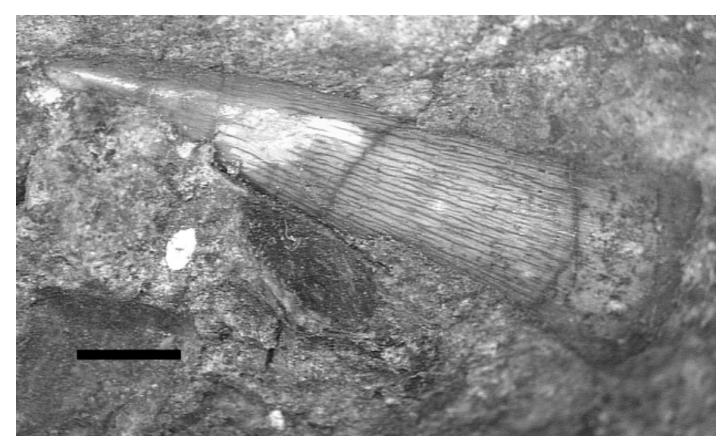

FIGURA 3 - Ictiodonte paleoniscóide curvo de grande porte (GP/2E-5973). Barra de escala: $2 \mathrm{~mm}$.

\section{DESCRIÇÃO DAS ESCAMAS}

4.1 Escamas lisas de superfície ondulada ou pontuada

Material - GP/2E-5954, GP/2E-5955, GP/ 2E-5956 e GP/2E-5958. Escamas isoladas.
Descrição - As escamas pontuadas nem sempre estão inteiras, mas são facilmente reconhecidas no pacote sedimentar. Este padrão é caracterizado pela superfície externa de ganoína, lisa ou levemente ondulada com alguns sulcos que seguem o contorno original da escama (Figuras 4A - D). Ocorrem poros (pontuações), que representam a abertura dos canais vasculares formados na camada de dentina e que atravessam as camadas de ganoína. Possuem forma rômbica, contorno liso, sem o processo articulatório "peg and socket" nos exemplares coletados, porém com parte da zona de imbricação preservada. O tamanho varia muito, tanto em comprimento, como em largura e espessura.

\subsection{Escamas costeladas}

Material - GP/2E-3757, GP/2E-3758, GP/ 2E-3759, GP/2E-3760, GP/2E-5959, GP/2E-5960. Escamas isoladas.

Descrição - As escamas costeladas são as mais facilmente reconhecidas nesse pacote sedimentar e também as mais abundantes. São caracterizadas pela superfície externa de ganoína com forma de diversas costelas longitudinais subparalelas a paralelas, que, em alguns exemplares, estão sobrepostas (Figuras 5A - F).

As margens normalmente são lisas, porém em muitos exemplares exibem aspecto serrilhado, devido à camada de ganoína que ultrapassa o limite da base articulatória (Figura 5A). Em escamas de margem não serrilhada, as costelas posicionam-se na parte central da escama (Figu$\operatorname{ras} 5 \mathrm{~B}, \mathrm{D}$ e $\mathrm{F})$.

A morfologia da região de imbricação de todas as escamas desse padrão é pouco conhecida devido ao desgaste, mas é possível observar boa
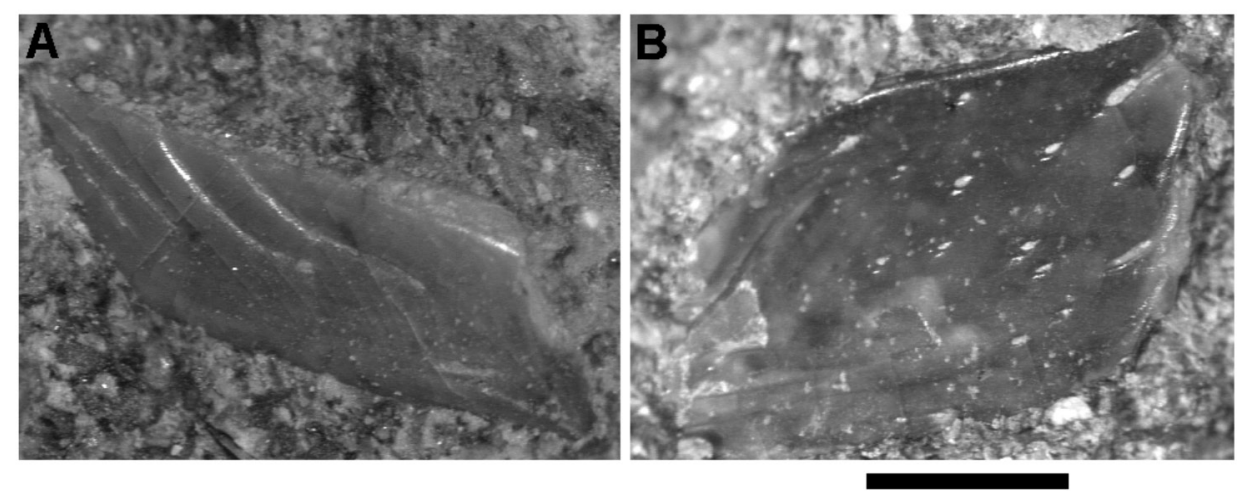

FIGURA 4 - Escamas lisas ou onduladas de paleoniscóides (GP/2E-5954, GP/2E-5956). Barra de escala: $1 \mathrm{~mm}$. 

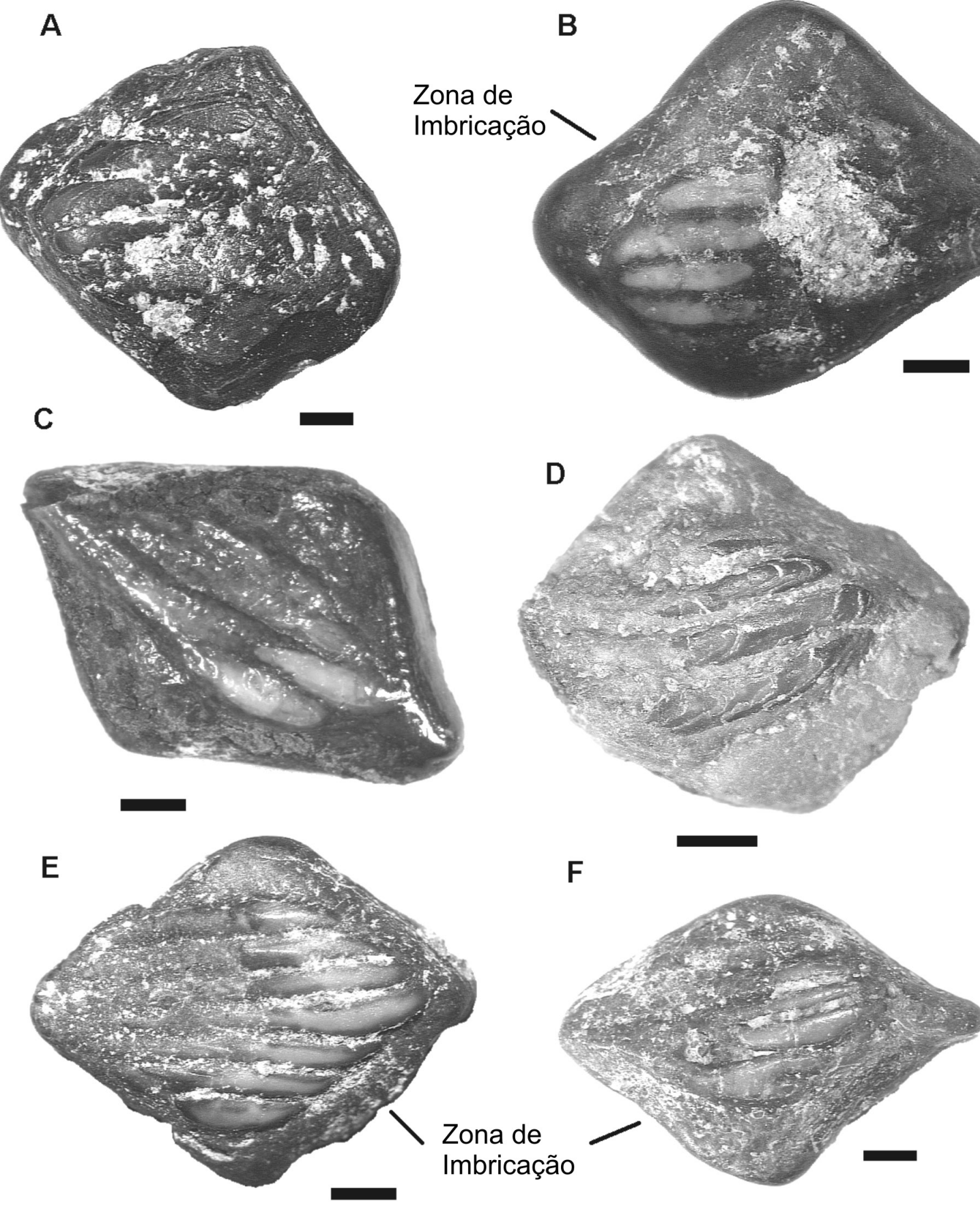

$\mathrm{F}$

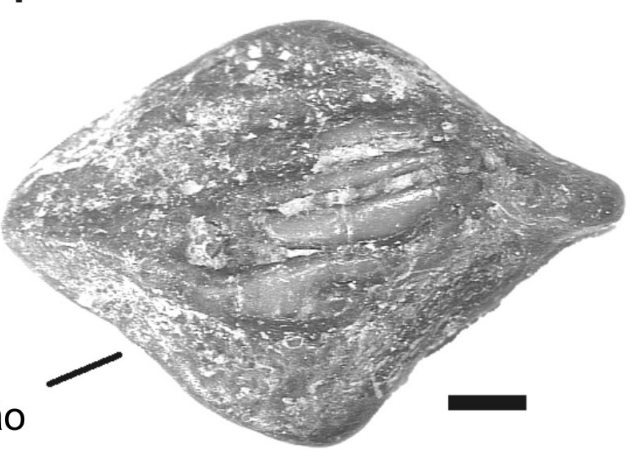

FIGURA 5 - Escamas costeladas de grande porte retiradas da matriz arenosa (GP/2E-3757, GP/2E-3758, GP/2E-3759, GP/2E-3760, GP/2E-5959, GP/2E-5960). Barra de escala: 2 mm. 
parte da margem correspondente da zona de imbricação em exemplares maiores (Figuras 5B, E e F). É caracterizada por faces lisas e extremidades que podem ser arredondadas ou angulosas. O comprimento das escamas varia de $0,5-15 \mathrm{~mm}$.

\subsection{Discussão dos exemplares}

RICHTER et al. (1985) foram as primeiras autoras a descrever o formato geral de escamas encontradas na Bacia do Paraná - Rio Grande do Sul, identificando cinco tipos de escamas ( $\mathrm{P}-1$ a $\mathrm{P}-5)$, correlacionando, com reservas, aos gêneros Acrolepis e Elonichthys. Como muitas escamas encontradas nos sedimentos grossos da base da Formação Irati no Estado de São Paulo estão desgastadas ou quebradas, as escamas foram classificadas em dois tipos bem diferenciados, escamas lisas com a superfície pontuada e escamas costeladas.

A partir da classificação de RICHTER et al. (1985), as escamas lisas de superfície pontuada podem ser consideradas como do tipo P - 1, enquanto as escamas costeladas podem ser associadas às do tipo $\mathrm{P}-2, \mathrm{P}-3, \mathrm{P}-4$ e $\mathrm{P}-5$.

As escamas estudadas possuem características externas muito semelhantes àquelas do material mais antigo do Neocarbonífero do Subgrupo Itararé (BRYANT 1929, BARCELLOS 1975), bem como do material mais recente do Neopermiano e Triássico da Bacia do Paraná (HUSSAKOF 1930, PEREZ \& MALABARBA 2002), sugerindo que os grupos de paleoniscóides pouco se modificaram do Carbonífero ao Triássico na Bacia do Paraná.

Além de escamas rômbicas foram encontradas formas irregulares que podem ser associadas a diversos ossos dérmicos e escamas fulcrais. $\mathrm{O}$ formato dessas partes ósseas e escamas varia de retangular a estirado ou alongado. SCHULTZE \& BARDACK (1987) estudaram essas "formas" diferenciadas em espécies do gênero Elonichthys provenientes do hemisfério norte e consideraram que muitas escamas retangulares ocorrem em partes de nadadeiras. A mesma observação foi feita por DUNKLE \& SCHAEFFER (1956) e RICHTER (2002) para espécimes completos da Bacia do Paraná.

\section{CONCLUSÕES}

A camada de arenito conglomerático da base da Formação Irati apresenta grande quantidade de fósseis de vertebrados, representados principalmente por escamas, dentes e ossos isolados de Os- teichthyes e, em segundo lugar, por dentes e raros espinhos de Chondrichthyes.

A maior parte dos ictiofósseis é representada por escamas lisas e costeladas e dentes retos, curvos e lisos. Escamas e dentes de paleoniscóides menores que $1 \mathrm{~cm}$ são comuns em todas as unidades estratigráficas do Carbonífero ao Triássico do Brasil que contêm ictiofósseis. Porém, grandes paleoniscóides com dentes e escamas de tamanho centimétrico são mais abundantes nas unidades permo-carboníferas e na base da Formação Irati (BARCELLOS 1975; COX \& HUTCHINSON 1991, CHAHUD 2003, 2007) e raras nas unidades neopaleozóicas e triássicas (HUSSAKOF 1930, WÜRDIG-MACIEL 1975, TOLEDO 2001, PEREZ \& MALABARBA 2002).

Dois tipos de ictiodontes descritos por RICHTER et al (1985) do Neopaleozóico do Rio Grande do Sul não foram observados na Formação Irati, porém dois novos morfotipos foram encontrados, os grandes dentes curvos e costelados com mais de um centímetro e um dente costelado apenas na região basal. As escamas observadas possuem características externas e de ornamentação como as do Neocarbonífero do Subgrupo Itararé (BRYANT 1929, BARCELLOS 1975) e do Neopermiano e Triássico da Bacia do Paraná (HUSSAKOF 1930, PEREZ \& MALABARBA 2002).

O caráter paleoambiental atribuído aos fósseis é incerto, pois os paleoniscóides são associados com vertebrados continentais e de água salgada.

\section{AGRADECIMENTOS}

Os autores expressam seu agradecimento ao Professor Doutor Thomas Rich Fairchild pelo apoio e auxílio dado em vários momentos durante essa pesquisa. Aos proprietários do Sítio Santa Maria no município de Rio Claro, Senhor Luis e Senhora Bernardete, onde foi desenvolvida a pesquisa. Ao Departamento de Geologia Sedimentar e Ambiental que permitiu que os trabalhos fossem realizados em seus laboratórios. Por último um agradecimento especial a CAPES e a FAPESP pelo apoio financeiro para o desenvolvimento da pesquisa.

\section{REFERÊNCIAS BIBLIOGRÁFICAS}

ASSINE, M.L.; ZACHARIAS, A.A.; PERINOTTO, J.A.J. 2003 Paleocorrentes, paleogeografia e seqüências deposicionais da Formação Tatuí, 
centro-leste do Estado de São Paulo. Revista Brasileira de Geociências, 33(1): 33-40.

BARCELLOS, M.T. 1975. Estudo de escamas e dentes de peixes da Fácies Budó, Sub-grupo Itararé, RGS. Boletim Paranaense de Geociências, 32: 3-65.

BRYANT, W.L. 1929. Fossil fish remains from the Permian tillite of Brazil. Bulletin Geological Society American, 40: 424-426.

CHAHUD, A. 2003. Paleoictiologia do topo da Formação Tatuí e base da Formação Taquaral (Subgrupo Irati), Permiano, Alto Estrutural de Pitanga, região de Rio Claro, SP. Instituto de Geociências, Universidade de São Paulo, São Paulo, Monografia de Trabalho de Formatura (Inédita), 49p.

CHAHUD, A. 2007. Paleontologia de Vertebrados da Transição entre os grupos Tubarão e Passa Dois no Centro-Leste do Estado de São Paulo. Instituto de Geociências, Universidade de São Paulo, São Paulo, 172p.

COX, C.B. \& HUTCHINSON, P. 1991. Fishes and amphibians from the Late Permian Pedra do Fogo Formation of Northern Brazil. Palaeontology, 34(3): 561-573.

DUNKLE, D.H. \& SCHAEFFER, B. 1956. Preliminary description of a paleoniscoid fish from the Late Paleozoic of Brazil. São Paulo: Faculdade de Filosofia, Ciências e Letras, Universidade de São Paulo, p. 5-22 (Boletim, Série Geologia, 13).

FIGUEIREDO, F.J. \& GALLO, V. 2006a. Actinopterígios paleopterígios: morfologia e taxonomia. In: V. Gallo; P. Brito; H. M. A. Silva; F. J. Figueiredo (eds.) Paleontologia de Vertebrados: Grandes Temas e Contribuições Científicas. Rio de Janeiro, Interciência, p. 1-24.

FIGUEIREDO, F.J. \& GALLO, V. 2006b. Uma história de actinopterígios paleopterígios. In: V. Gallo; P. Brito; H. M. A. Silva; F. J. Figueiredo. (eds.) Paleontologia de Vertebrados: Grandes Temas e Contribuições Científicas. Rio de Janeiro, Interciência, p. 25-46.

FÚLFARO, V.J.; STEVAUX, J.C.; SOUZA-FILHO, E.E.; BARCELOS, J.H. 1984. A Formação Tatuí (P) no Estado de São Paulo. In: SBG, CONGRESSO BRASILEIRO DE
GEOLOGIA, 23, Rio de Janeiro, Anais, 2: 711-723.

HACHIRO, J. 1997 O Subgrupo Irati (Neopermiano) da Bacia do Paraná. Instituto de Geociências, Universidade de São Paulo, São Paulo, Tese de Doutoramento, 196p.

HUSSAKOF, L. 1930. Alguns restos de peixes do Permiano e do Triássico do Brasil. Serviço Geológico e Mineralógico do Brasil, p. 1-11. (Boletim, 49).

LONG, J.A. 1993. Morphological characteristics of Palaeozoic vertebrates used in biostratigraphy. In: J.A. LONG (ed.) Palaeozoic vertebrate biostratigraphy and biogeography. London Belhaven, p. 3-24.

LONG, J.A. 1995. The Rise of Fishes: 500 Million Years of Evolution. University of New South Wales Press, Sydney, 223p.

MARANHÃO, M.S.A.S. 1995. Fósseis das Formações Corumbataí e Estrada Nova do Estado de São Paulo: Subsídios ao conhecimento Paleontológico e Bioestratigráfico. Instituto de Geociências, Universidade de São Paulo, São Paulo, Tese de Doutoramento, 2 v.

PEREZ, M.A. \& MALABARBA, M.C.S.L. 2002. A Triassic freshwater fish fauna from the $\mathrm{Pa}-$ raná Basin, in southern Brazil. Revista Brasileira de Paleontologia, 4: 27-33.

RAGONHA, E.W. 1978. Chondrichthyes do Membro Taquaral (Formação Irati) no Estado de São Paulo. Instituto de Geociências, Universidade de São Paulo, São Paulo, Dissertação de Mestrado, 65p.

RICHTER, M. 2002. A ray-finned fish (Osteichthyes) from the Late Permian of the State of Santa Catarina (Paraná Basin), Southern Brazil. Revista Brasileira de Paleontologia, 3: 56-61.

RICHTER, M.; PICCOLI, A.M.M.; LIMA, M.C.S. 1985. Variação morfológica de restos de paleoniscídeos (Pisces) no Permiano da Bacia do Paraná. In: DNPM, Coletânea de Trabalhos Paleontológicos, Brasília, Série Geologia (27), Seção de Paleontologia e Estratigrafia (2), p. 111-122. 
RICHTER, M.; VIANA, M.S.S. \& MALABARBA, M.C.S.L. 2004. Agnatos e Peixes. In: I.S. CARVALHO (ed.) Paleontologia. Rio de Janeiro, Editora Interciência, 1:733-761.

SANTOS, R.V.; SOUZA, P.A. ALVARENGA, C.J.S.; DANTAS, E.L.; PIMENTEL, E.L.; OLIVEIRA, C.G.; ARAÚJO, L.M. 2006. Shrimp U-Pb Zircon Dating and Palynology of Bentonitic Layers from the Permian Irati Formation Parana Basin, Brazil. Gondwana Research, 9: 456-463.

SCHULTZE, H. P. \& BARDACK, D. 1987. Diversity and size changes in palaeonisciform fishes (Actinopterygii, Pisces) from the Pannsylvanian Mazon Creek Fauna, Illinois, U.S.A. Journal of Vertebrate Paleontology, 7(1): 1-23.
STEVAUX, J.C.; SOUZA-FILHO, E. E. \& FÚLFARO, V.J. 1986. Trato deposicional da Formação Tatuí (P) na área aflorante do NE da Bacia do Paraná, Estado de São Paulo. In: SBG, CONGRESSO BRASILEIRO DE GEOLOGIA, 34. Goiânia, Anais, 1: 219-229.

TOLEDO, C. E. V. 2001. Análise paleontológica da Formação Corumbataí na Região de Rio Claro, Estado de São Paulo. Instituto de Geociências e Ciências Exatas, UNESP, Rio Claro, Dissertação de Mestrado, 146p.

WÜRDIG-MACIEL, N.L. 1975. Ichtiodontes e ichtiodorulitos (Pisces) da Formação Estrada Nova e sua aplicação na estratigrafia do Grupo Passa Dois. Pesquisas, 5:7-165.

Endereço dos autores:

Artur Chahud - Departamento de Geologia Sedimentar e Ambiental, Instituto de Geociências, Universidade de São Paulo, Rua do Lago, 562, CEP 05508-900, Cidade Universitária, São Paulo, SP. E-mail: arturchahud@yahoo.com

Setembrino Petri - Departamento de Geologia Sedimentar e Ambiental, Instituto de Geociências, Universidade de São Paulo, Rua do Lago, 562, CEP 05508-900, Cidade Universitária, São Paulo, SP. E-mail: spetri@usp.br 